\title{
G-CSF producing oral carcinoma with diffuse uptake of FDG in the bone marrow: A case report
}

\author{
TAKESHI KUROSHIMA ${ }^{1}$, MAYUMI WADA ${ }^{2}$, TAKEHIKO SATO ${ }^{2}$, \\ MASASHI TAKANO ${ }^{2}$ and SHUJIROH MAKINO ${ }^{2}$ \\ ${ }^{1}$ Oral and Maxillofacial Surgery, Department of Oral Restitution, Division of \\ Oral Health Sciences, Graduate School of Medical and Dental Sciences, Tokyo Medical and Dental University, \\ Tokyo 113-8549; ${ }^{2}$ Department of Oral Surgery, Hokuto Hospital, Obihiro, Hokkaido 080-0833 Japan
}

Received July 26, 2017; Accepted November 6, 2017

DOI 10.3892/ol.2017.7422

\begin{abstract}
A 78-year-old male patient was referred to the Department of Oral Surgery, Hokuto Hospital (Obihiro, Japan) for painless swelling on the left neck and tongue. Histopathological examination of a biopsy specimen resulted in a diagnosis of squamous cell carcinoma of the tongue. Imaging examinations revealed cervical lymph node metastases on both sides, along with diffuse uptake of ${ }^{18} \mathrm{~F}$-fluorodeoxyglucose (FDG) in the bone marrow of the spine and pelvis. Hematologic tests revealed an increased white blood cell (WBC) count and serum concentrations of granulocyte colony stimulating factor (G-CSF). These findings suggested a G-CSF producing tumor, with fluctuations of WBC count, serum G-CSF concentration, and FDG uptake in the bone marrow, associated with tumor shrinkage and enlargement, an indicator of tumor status.
\end{abstract}

\section{Introduction}

Granulocyte colony stimulating factor (G-CSF) is a cytokine mainly produced by macrophages, fibroblasts and endothelial cells in an inflammatory milieu. G-CSF stimulates neutrophil precursors, resulting in an increase in neutrophils, and recruits neutrophils from the bone marrow to peripheral blood. G-CSF is an important factor in infection prophylaxis, and recombinant G-CSF is universally used to treat neutropenia (1). G-CSF is also produced by non-hematologic malignancies with high leukocyte counts, consisting predominantly of neutrophils, in patients without infectious diseases. Most of these G-CSF producing tumors are present in the lungs (2), with tumors

Correspondence to: Dr Takeshi Kuroshima, Oral and Maxillofacial Surgery, Department of Oral Restitution, Division of Oral Health Sciences, Graduate School of Medical and Dental Sciences, Tokyo Medical and Dental University, 1-5-45 Yushima, Bunkyo-ku, Tokyo 113-8549, Japan

E-mail: kuroosur@tmd.ac.jp

Key words: G-CSF producing tumor, oral cancer, FDG-PET, bone marrow in the oral regions being rare. This report describes a patient with a tongue carcinoma producing G-CSF, as well as showing diffuse uptake of FDG in the bone marrow.

\section{Case report}

In July 2013, a 78-year-old man visited the Department of Oral Surgery, Hokuto Hospital (Obihiro, Japan) with a 2-week history of painless swelling on the left neck and tongue. The patient had no systemic complications and no significant family history. Some cervical lymph nodes on both sides were palpable (Fig. 1A). Intra-oral examination showed a tumor with induration about $40 \mathrm{~mm}$ in diameter on the left side of the tongue (Fig. 1B). Cytological examination of a swollen left lymph node showed atypical squamous cells, and pathological examination of a biopsy of the tongue tumor revealed a squamous cell carcinoma. A computed tomography (CT) scan with contrast demonstrated a large lateral oral tongue tumor of diameter $42 \mathrm{~mm}$, without extension to the extrinsic muscles of the tongue; and some metastatic cervical lymph nodes that were enlarged, nonhomogeneously enhanced, and partially necrotic. Metastatic disease of left middle jugular lymph node was $>30 \mathrm{~mm}$ in maximum diameter (Fig. 2). ${ }^{18} \mathrm{~F}$-fluorodeoxyglucose-positron emission tomography (FDG-PET)/CT showed abnormally high uptake by the tongue tumor (maximum standardized uptake value [SUVmax] 22.19) and by the four large metastatic nodes, with the large left middle jugular node having an SUVmax of 14.43 (Fig. 3A). Diffuse FDG uptake was also observed in the bone marrow of the spine and pelvis (Fig. 3B). These findings suggested that hematopoietic capacity was enhanced. Hematological examination showed leukocytosis (WBC count 21,680 $\mu \mathrm{l}^{-1}$ ), dominated by neutrophils (86.4\%), and a high serum concentration of C-reactive protein (CRP), $4.47 \mathrm{mg} / \mathrm{dl}$ (Table I). Because we suspected that these findings were due to G-CSF produced by the tumor, additional analyses were performed. Although immunohistochemical staining of a paraffin-embedded tongue biopsy specimen with monoclonal anti-G-CSF antibody yielded negative results, the patient's serum G-CSF level was increased, to $117.0 \mathrm{pg} / \mathrm{ml}$ (normal, <39.0 pg/ml). He had no symptoms of infectious disease, including fever, and no blast cells in his peripheral blood. 
Because the initial diagnosis of the tumor was cT3N3M0, we thought that it was resectable. However, the patient refused surgical treatment, radiation therapy and intravenous chemotherapy. Therefore, he was treated with oral chemotherapy, consisting of 3-week cycles of $100 \mathrm{mg} /$ day $\mathrm{S} 1$ for 2 weeks followed by a 1 -week rest. Following the second treatment cycle, we observed marked shrinkage of the patient's primary tumor and metastatic cervical lymph nodes (Fig. 4), along with reductions in his WBC count $\left(9,930 \mu \mathrm{l}^{-1}\right)$, neutrophil count $(72.7 \%)$ and serum CRP $(0.47 \mathrm{mg} / \mathrm{dl})$ and G-CSF (54.9 pg/ml) concentrations (Fig. 5). After the fourth cycle of chemotherapy, the tongue tumor further decreased in size (Fig. 6), but regrowth of the tumor in the left middle jugular node was observed. In addition, his WBC count $\left(13,670 \mu \mathrm{l}^{-1}\right)$, neutrophil count $(79.2 \%)$ and CRP concentration $(1.19 \mathrm{mg} / \mathrm{dl})$ had again increased (Fig. 5). At the end of the sixth cycle of chemotherapy, the tumor in the left node had increased further and become painful, and his WBC count $\left(24,980 \mu \mathrm{l}^{-1}\right)$, neutrophil count (87.2\%) and CRP concentration $(3.85 \mathrm{mg} / \mathrm{dl}) \mathrm{had}$ increased (Fig. 5). Although FDG uptake was observed in the left metastatic node (SUVmax 17.52) and the bone marrow, FDG did not accumulate in the primary tongue tumor and in other node lesions (Fig. 7). Because of neck pain, the patient consented to chemoradiotherapy as curative treatment with the possibility of pain relief. He was therefore started on intensity-modulated radiation therapy with concurrent low-dose daily CDDP $\left(4 \mathrm{mg} / \mathrm{m}^{2}\right.$ ), but radiation was discontinued at $10 \mathrm{~Gy}$ due to patient refusal. Thereafter, he received supportive care and died of the disease one year after initial examination.

\section{Discussion}

Leukocytosis in association with non-hematologic malignancies in the absence of infectious disease is a paraneoplastic syndrome (1). These tumors are thought to produce G-CSF, resulting in leukocytosis, consisting predominantly of neutrophils. G-CSF-producing tumors are characterized by i) increased WBC counts, predominantly neutrophils, in the absence of infectious and hematologic diseases; ii) increased serum G-CSF level; iii) normalization of WBC count and serum G-CSF level after remission or removal of the tumor; and iv) presence of G-CSF in tumor tissues $(3,4)$.

At initial examination, the patient described in this report was found to have marked leukocytosis, predominantly consisting of neutrophils, and increased serum G-CSF levels. Immunohistochemical staining of a tongue biopsy specimen with monoclonal anti-G-CSF antibody yielded negative results, similar to findings in many other patients with G-CSF producing tumors $(5,6)$. Negative staining of tumor tissue may be caused by the rapid secretion of G-CSF from tumor cells (7). Although this tumor was not resected, oral chemotherapy, which reduced the size of the tumor, reduced his WBC count and serum G-CSF concentration to near normal levels. S1 can cause hematotoxicity, resulting in decreased WBC count. Therefore, the reductions of WBC count and serum G-CSF concentration in our patient may have been related to the side effects caused by $\mathrm{S} 1$. However, subsequent regrowth of the tumor on the left node was accompanied by increases in WBC count and serum G-CSF concentration. The correlation of these two parameters with shrinkage and
A

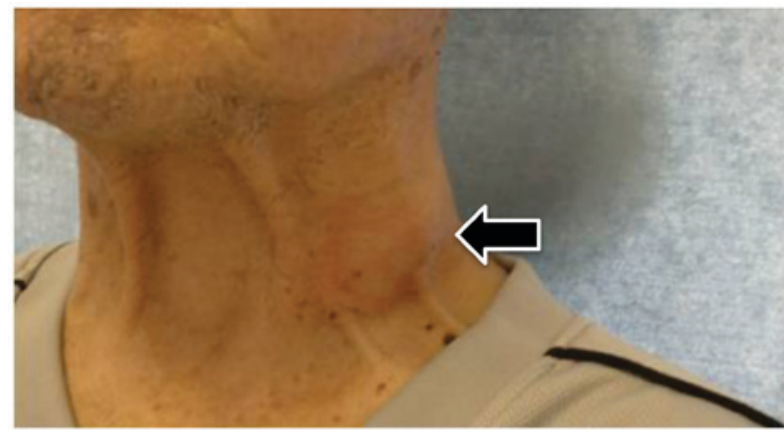

$\mathrm{B}$

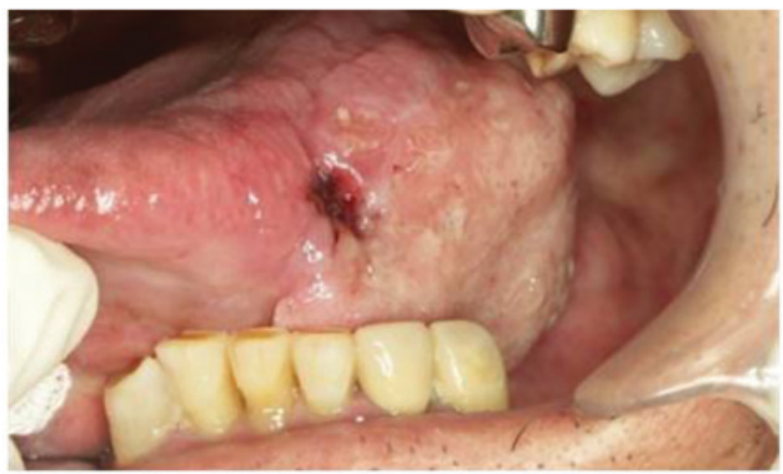

Figure 1. Physical findings at first examination. (A) A swollen node on the left side of the neck. (B) The tumor on the left side of the tongue.

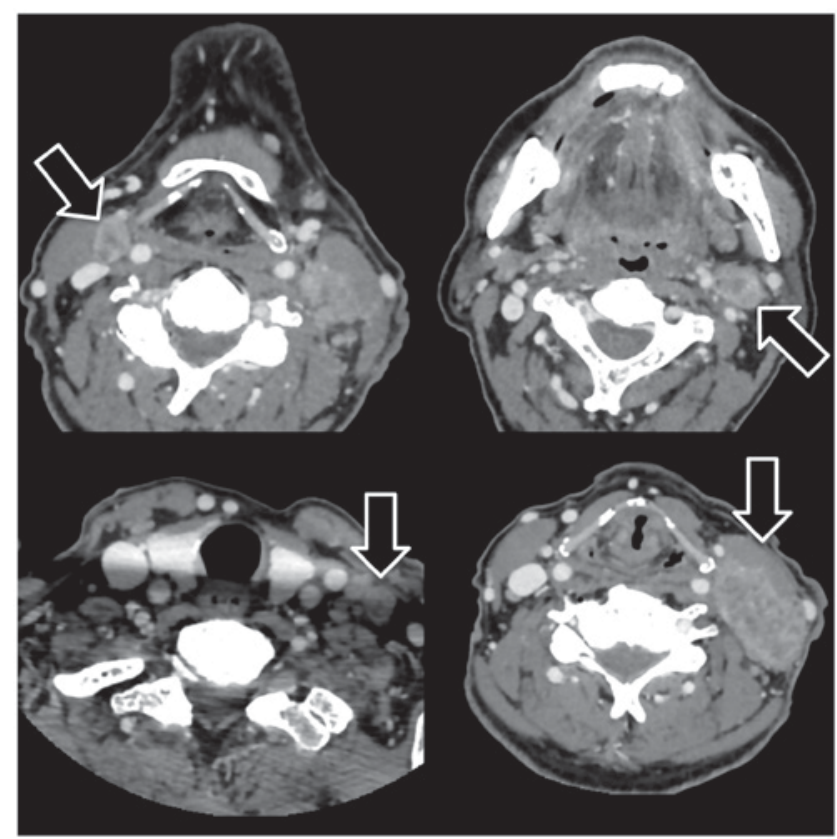

Figure 2. Axial contrast-enhanced computed tomography scan showing four enlarged, necrotic nodes, with irregular and enhancing rims on both sides of each.

enlargement of the tumor suggested that this tumor produced G-CSF.

FDG-PET, which assesses glucose uptake and metabolism, is broadly recognized as a useful modality for tumor imaging. FDG-PET imaging of patients with G-CSF-producing tumors has shown diffuse uptake of FDG throughout the bone marrow and markedly elevated uptake of FDG by the primary tumor (2). Moreover, the bone marrow uptake of FDG was 

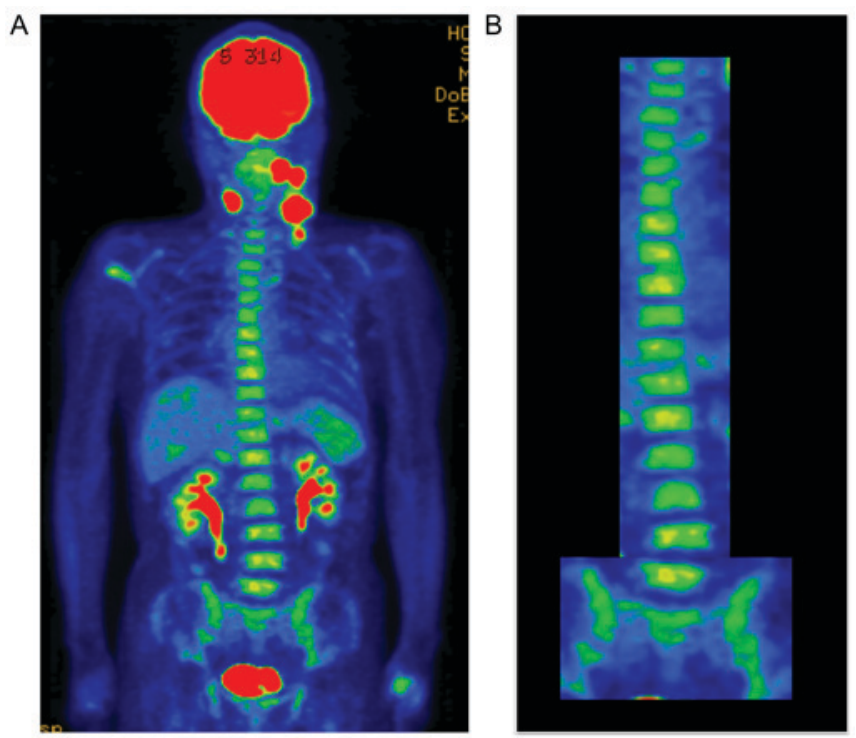

Figure 3. FDG-PET findings before chemotherapy. (A) High uptake of FDG by the tumor on the left side of the tongue and the node lesions. (B) Diffuse uptake of FDG by bone marrow. FDG-PET, 18F-fluorodeoxyglucose-positron emission tomography.

A
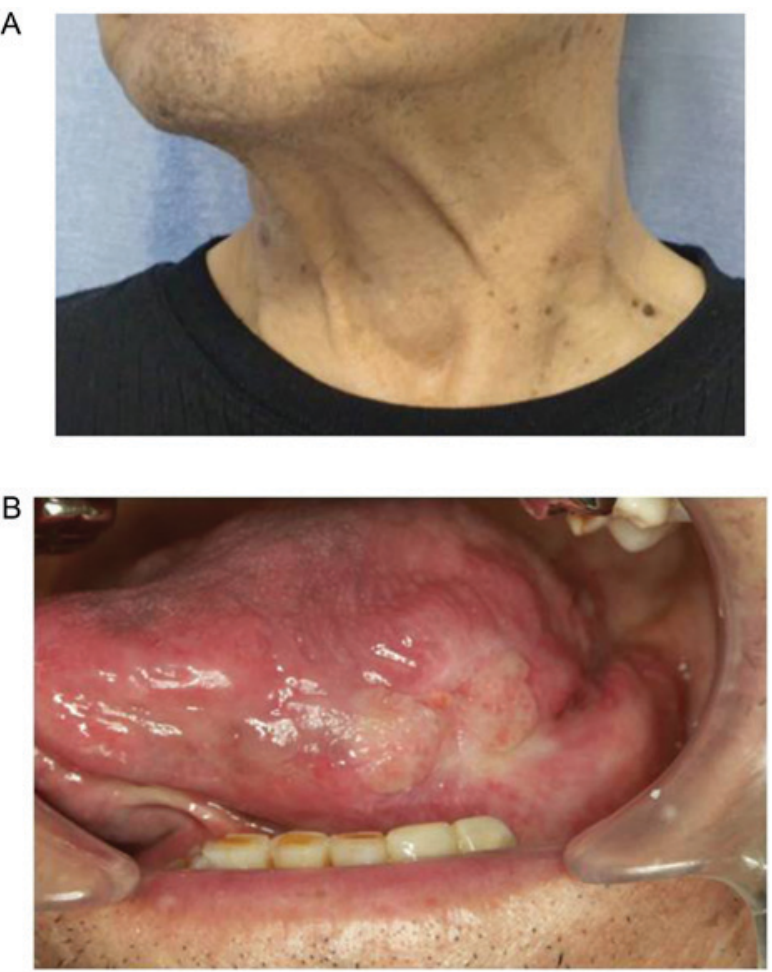

Figure 4. Physical findings following the second cycle of S1 treatment. (A) Marked reduction in the size of the node lesions. (B) Marked shrinkage of the primary tongue tumor.

found to correlate with peripheral WBC count, especially neutrophils, and to reflect the increased metabolic activity of bone marrow (8). Treatment with recombinant G-CSF has been reported to increase the metabolism and cellularity of bone marrow, resulting in increased bone marrow uptake of FDG (9). Thus, G-CSF producing tumors are thought to enhance bone marrow metabolism, resulting in FDG uptake in the bone marrow.
Table I. Hematological examination at first visit.

\begin{tabular}{|c|c|}
\hline Variables & Values \\
\hline WBC & $21,680 / \mu 1$ \\
\hline Neut & $86.4 \%$ \\
\hline Eos & $1.0 \%$ \\
\hline Bas & $0.2 \%$ \\
\hline Mon & $4.2 \%$ \\
\hline Lym & $8.2 \%$ \\
\hline $\mathrm{RBC}$ & $297 \times 10^{4} / \mu 1$ \\
\hline $\mathrm{Hb}$ & $9.0 \mathrm{~g} / \mathrm{dl}$ \\
\hline Hct & $27.4 \%$ \\
\hline Plt & $44.8 \times 10^{3} / \mu 1$ \\
\hline $\mathrm{TP}$ & $6.7 \mathrm{~g} / \mathrm{dl}$ \\
\hline Alb & $2.7 \mathrm{~g} / \mathrm{dl}$ \\
\hline AST & $12 \mathrm{U} / 1$ \\
\hline ALT & $12 \mathrm{U} / 1$ \\
\hline $\mathrm{LDH}$ & $109 \mathrm{U} / 1$ \\
\hline$\gamma$-GTP & $33 \mathrm{U} / 1$ \\
\hline BUN & $8.9 \mathrm{mg} / \mathrm{dl}$ \\
\hline $\mathrm{Cr}$ & $0.67 \mathrm{mg} / \mathrm{dl}$ \\
\hline $\mathrm{Na}$ & $136 \mathrm{mEq} / \mathrm{l}$ \\
\hline $\mathrm{K}$ & $4.0 \mathrm{mEq} / 1$ \\
\hline $\mathrm{Cl}$ & $102 \mathrm{mEq} / 1$ \\
\hline $\mathrm{Ca}$ & $7.8 \mathrm{mEq} / \mathrm{l}$ \\
\hline CRP & $4.5 \mathrm{mg} / \mathrm{dl}$ \\
\hline SCC & $1.3 \mathrm{ng} / \mathrm{ml}$ \\
\hline G-CSF & $117.0 \mathrm{pg} / \mathrm{ml}$ \\
\hline
\end{tabular}

WBC, white blood cells; Neut, neutrophils; Eos, Eosinophils; Mon, monocytes; Lym, lymphocytes; RBC, red blood cells; Hb, hemoglobin; Hct, hematocrit; Plt, platelets; TP, total protein; Alb, albumin; AST, aspartate aminotransferase; ALT, alanine aminotransferase; LDH, lactate dehydrogenase; $\gamma$-GTP, $\gamma$-glutamyl transpeptidase; BUN, blood urea nitrogen; $\mathrm{Cr}$, creatinine; $\mathrm{Na}$, sodium; $\mathrm{K}$, potassium; $\mathrm{Cl}$, Chloride; $\mathrm{Ca}$, calcium; CRP, C-reactive protein; $\mathrm{SCC}$, squamous cell carcinoma antigen; G-CSF, granulocyte colony stimulating factor.

Cancer patients with FDG-uptake in the bone marrow require differential diagnosis of bone metastases. In general, bone metastases from oral squamous cell carcinoma, a solid tumor type, are present as solid tumors with focal uptake of FDG. In contrast, the diffuse uptake of FDG in the bone marrow has also been reported in patients with leukemia, lymphoma, histiocytosis, myeloma, and myeloid hyperplasia, as well as after treatment with cytokines and erythropoietin (10). In other words, diffuse uptake of FDG in bone marrow does not indicate metastases from oral cancer, in general. In our patient, uptake of FDG in the bone marrow was not focal but diffuse. Therefore, we deemed that there was no metastasis to the bone marrow and that diffuse uptake of FDG in the bone marrow was founded to reflect the increased metabolic activity of bone marrow by G-CSF. Moreover, our patient had no symptoms or laboratory findings suggesting that bone marrow disease or hematopoietic malignancy was the cause of diffuse uptake of FDG in bone 


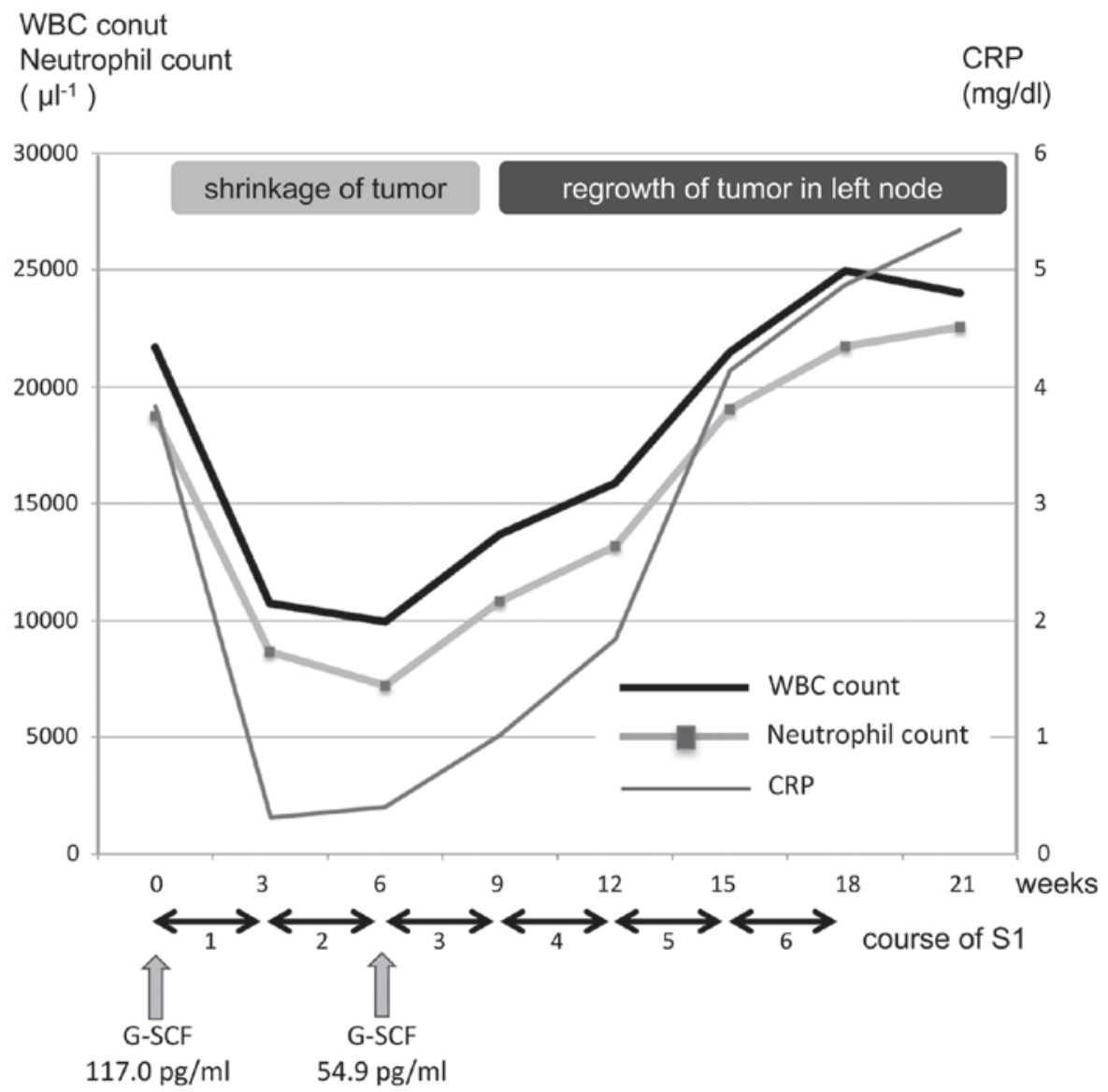

Figure 5. Laboratory findings during the course of chemotherapy. Following the second cycle of S1, WBC count, neutrophil count and serum G-CSF and CRP concentrations were decreased to near normal, with shrinkage of the primary tumor and metastatic nodes. Following the fourth cycle, WBC count, neutrophil count and CRP increased with regrowth of the tumor in the left node. WBC, white blood cells; G-CSF, granulocyte colony stimulating factor; CRP, C-reactive protein.

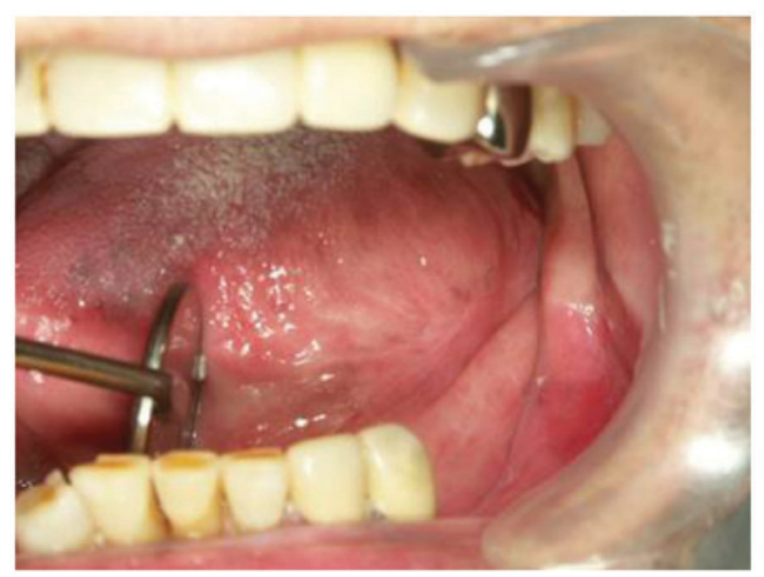

Figure 6. View showing disappearance of the tongue tumor after the fourth cycle of S1 treatment.

marrow. Therefore, we did not perform a bone marrow examination in this patient.

G-CSF produced by a tumor may also contribute to marked tumor infiltration by inflammatory cells, a process that which may enhance tumor uptake of FDG (2). Our patient showed marked FDG uptake by both the tongue tumor and the metastatic nodes. In addition, the regrown tumor in the left node showed marked FDG uptake, with an SUVmax of
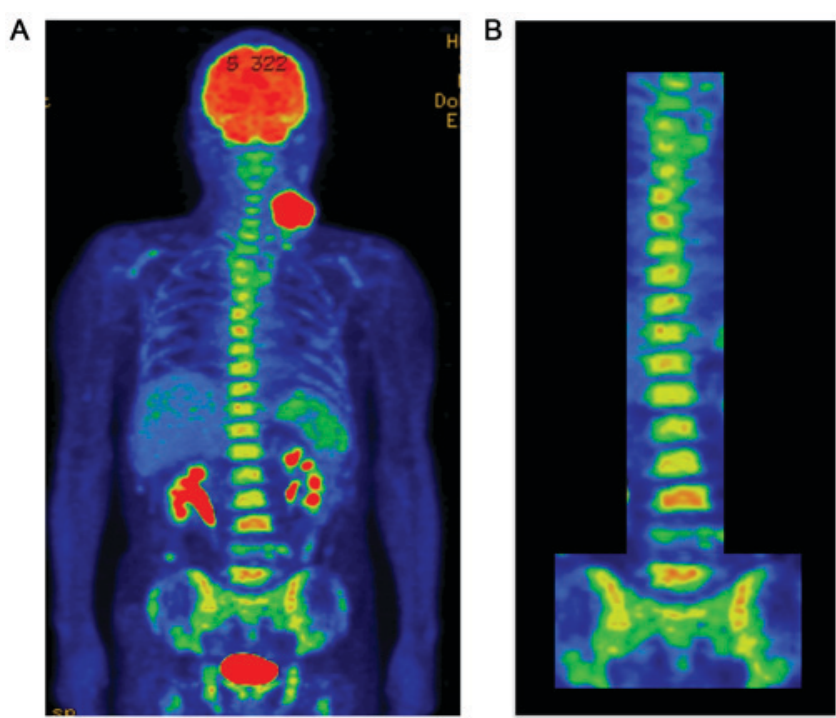

Figure 7. FDG-PET findings after chemotherapy. (A) Uptake of FDG by the primary tongue tumor and some node lesions was not observed. However, the left metastatic node showed high uptake of FDG. (B) Diffuse uptake of FDG by bone marrow. FDG-PET, 18F-fluorodeoxyglucose-positron emission tomography.

17.52, despite the absence of uptake by the primary tongue tumor and the other metastatic nodes. WBC count, CRP 
concentration and FDG uptake in bone marrow were also increased at the same time. These findings suggested that the tumor in the left node, rather than the primary tongue tumor and the other metastatic nodes, may have the potential to produce $\mathrm{G}-\mathrm{CSF}$.

G-CSF producing tumors are associated with aggressive tumor behavior and poor patient prognosis, regardless of the primary site and pathological type of the tumor. The lungs are the most frequent site of G-CSF producing tumors (2), followed by sites such as the liver (11), stomach (5) and bladder (1). However, G-CSF producing tumors are rarely detected in the oral regions $(4,7,12,13)$. The survival time of patients with G-CSF-producing tumors has been reported to be approximately 6 months (5). To our knowledge, 7 of 8 patients with these tumors in the head and neck region died within 1 year of diagnosis (average 8 months), as did our patient $(4,7,12-17)$.

Several hypotheses may explain the causes poor prognosis of patients with G-CSF-producing tumors. G-CSF is frequently produced by poorly differentiated and undifferentiated carcinomas $(5,6)$, and many of these G-CSF producing tumors are initially detected at advanced stage $(1,5-7,12,13)$. In addition, the G-CSF produced by these tumors may contribute to tumor progression by autocrine and paracrine mechanisms $(18,19)$, as well as promoting angiogenesis and tumor growth (20). No standard therapy has yet been established for G-CSF producing tumors, but multimodal treatment should be initiated rapidly to improve prognosis.

In conclusion, although oral G-CSF producing tumors are rare, the combination of diffuse uptake of FDG in the bone marrow and leukocytosis, especially of neutrophils, may suggest this tumor type. Alterations in these parameters may be useful as markers of tumor status.

\section{Acknowledgements}

This study was partially supported by JSPS KAKENHI (Grant no. 6K20433).

\section{References}

1. Kumar AK, Satyan MT, Holzbeierlein J, Mirza M and Van Veldhuizen P: Leukemoid reaction and autocrine growth of bladder cancer induced by paraneoplastic production of granulocyte colony-stimulating factor-a potential neoplastic marker: A case report and review of the literature. J Med Case Rep 8: 147,2014

2. Morooka M, Kubota K, Murata Y, Shibuya H, Ito K, Mochizuki M, Akashi T, Chiba T, Nomura T, Ito H and Morita T: (18)F-FDG-PET/CT findings of granulocyte colony stimulating factor (G-CSF)-producing lung tumors. Ann Nucl Med 22: 635-639, 2008.

3. Asano S, Urabe A, Okabe T, Sato N and Kondo Y: Demonstration of granulopoietic factor(s) in the plasma of nude mice transplanted with a human lung cancer and in the tumor tissue. Blood 49: 845-852, 1977.
4. Kobayashi J, Miyazaki A, Yamamot T, Nakamori K, Suzuki R, Kaneko T, Suzuki N, and Hiratsuka H: Granulocyte colony-stimulating factor-producing squamous cell carcinoma of the lower gingiva: A case report. Head Neck Oncol 4: 35, 2012.

5. Kawaguchi M, Asada Y, Terada T, Takehara A, Munemoto Y, Fujisawa K, Mitsui T, Iida Y, Miura S and Sudo Y: Aggressive recurrence of gastric cancer as a granulocyte-colony-stimulating factor-producing tumor. Int J Clin Oncol 15: 191-195, 2010.

6. Vinzens S, Zindel J, Zweifel M, Rau T, Gloor B and Wochner A: Granulocyte colony-stimulating factor producing anaplastic carcinoma of the pancreas: Case report and review of the literature. Anticancer Res 37: 223-228, 2017.

7. Horii A, Shimamura K, Honjo Y, Mitani K, Miki T, Takashima S and Yoshida J: Granulocyte colony stimulating factor-producing tongue carcinoma. Head Neck 19: 351-356, 1997.

8. Murata Y, Kubota K, Yukihiro M, Ito K, Watanabe H and Shibuya H: Correlations between 18F-FDG uptake by bone marrow and hematological parameters: Measurements by PET/CT. Nucl Med Biol 33: 999-1004, 2006.

9. Sugawara Y, Zasadny KR, Kison PV, Baker LH and Wahl RL: Splenic fluorodeoxyglucose uptake increased by granulocyte colony-stimulating factor therapy: PET imaging results. J Nucl Med 40: 1456-1462, 1999.

10. Goshen E, Davidson T, Yeshurun M and Zwas ST: Combined increased and decreased skeletal uptake of F-18 FDG. Clin Nucl Med 31: 520-522, 2006.

11. Nagata H, Komatsu S, Takaki W, Okayama T, Sawabe Y, Ishii M, Kishimoto M, Otsuji E and Konosu H: Granulocyte colony-stimulating factor-producing hepatocellular carcinoma with abrupt changes. World J Clin Oncol 7: 380-386, 2016.

12. Kaneko N, Kawano S, Matsubara R, Goto Y, Jinno T, Maruse Y, Sakamoto T, Hashiguchi Y, Iida M and Nakamura S: Tongue squamous cell carcinoma producing both parathyroid hormone-related protein and granulocyte colony-stimulating factor: A case report and literature review. World J Surg Oncol 14: $161,2016$.

13. Ishigami T, Yoshida H, Yusa H and Yanagawa T: Gingival cancer suspected of producing granulocyte colony-stimulating factor: Report of a case. J Oral Maxillofac Surg 59: 804-808, 2001.

14. Yazawa S, Toshimori H, Nakatsuru K, Katakami H, Takemura J and Matsukura S: Thyroid anaplastic carcinoma producing granulocyte-colony-stimulating factor and parathyroid hormone-related protein. Intern Med 34: 584-588, 1995.

15. Yoneda T, Nishimura R, Kato I, Ohmae M, Takita M and Sakuda M: Frequency of the hypercalcemia-leukocytosis syndrome in oral malignancies. Cancer 68: 617-622, 1991.

16. Tanaka $K$ and Nibu K: Laryngeal squamous cell carcinoma with ectopic production of granulocyte colony-stimulating factor and parathyroid hormone-related protein. Int J Clin Oncol 10: 195-197, 2005.

17. Tamura K, Yoshinaga T, Tanino M, Kimura T, Yamada N, Nishimura M, Fukuda S, Nishihara H, Shindoh $M$ and Tanaka S: Hypopharyngeal squamous cell carcinoma producing both granulocyte colony-stimulating factor and parathyroid hormone-related protein. Pathol Int 58: 652-656, 2008.

18. Inoue M, Minami M, Fujii Y, Matsuda H, Shirakura R and Kido T: Granulocyte colony-stimulating factor and interleukin-6-producing lung cancer cell line, LCAM. J Surg Oncol 64: 347-350, 1997.

19. Ichiishi E, Yoshikawa T, Kogawa T, Yoshida N and Kondo M: Possible paracrine growth of adenocarcinoma of the stomach induced by granulocyte colony stimulating factor produced by squamous cell carcinoma of the oesophagus. Gut 46: 432-434, 2000.

20. Natori T, Sata M, Washida M, Hirata Y,Nagai R and Makuuchi M: G-CSF stimulates angiogenesis and promotes tumor growth: Potential contribution of bone marrow-derived endothelial progenitor cells. Biochem Biophys Res Commun 297: 1058-1061, 2002. 